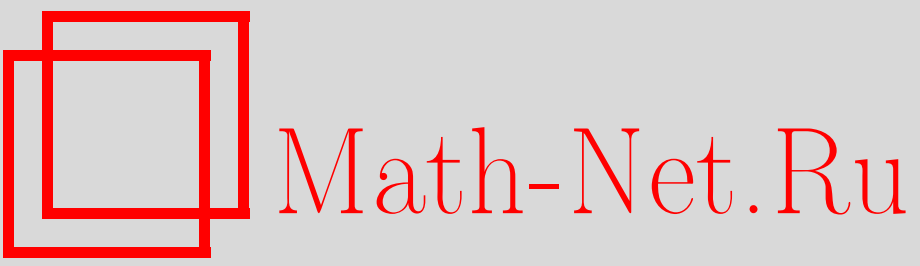

С. Ю. Немировский, Комплексный анализ и дифференциальная топология на комплексных поверхностях, УМН, 1999, том 54, выпуск 4, 47-74

DOI: https://doi.org/10.4213/rm179

Использование Общероссийского математического портала Math-Net.Ru подразумевает, что вы прочитали и согласны с пользовательским соглашением

http://www.mathnet.ru/rus/agreement

Параметры загрузки:

IP: 3.89.197.203

26 апреля 2023 г., 13:52:09 


\title{
КОМПЛЕКСНЫЙ АНАЛИЗ И ДИФФЕРЕНЦИАЛЬНАЯ ТОПОЛОГИЯ НА КОМПЛЕКСНЫХ ПОВЕРХНОСТЯХ
}

\author{
С. Ю. НЕмиРОвСкий
}

\begin{abstract}
В статье описывается связь между теорией голоморфных функций на двумерных комплексных многообразиях и их дифференциальной топологией. Ключевое утверждение, получаемое с помощью инвариантов Зайберга-Виттена, заключается в том, что топологические характеристики вложенных вещественных двумерных поверхностей в штейновых комплексных поверхностях удовлетворяют неравенствам типа неравенства присоединения. Вариант $h$-принципа М. Громова для вполне вещественных вложений позволяет показать, что эти неравенства точны. В ряде случаев эти резултаты можно использовать для описания оболочек голоморфности вложенных вещественных поверхностей в данной комплексной поверхности. В работе рассмотрены вещественные поверхности в $\mathbb{C}^{2}, \mathbb{C P}^{2}$ и в произведениях $\mathbb{C P}^{1}$ на некомпактные римановы поверхности. Аналогичный метод применим к изучению геометрических свойств двумерных строго псевдовыпуклых областей.
\end{abstract}

Библиографияя: 44 названия.

\section{СОДЕРЖАНИЕ}

$\S 1$. Оболочки голоморфности и проблема Леви

$\S 2$. Комплексные точки и штейновы окрестности вещественных поверх-

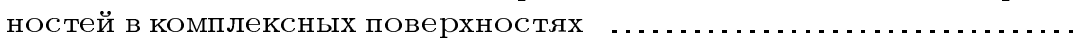

$\S$ 3. Инварианты Зайберга-Виттена и неравенства для рода вложенных

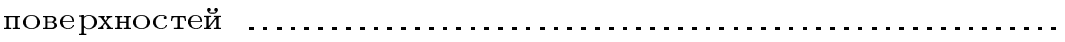

$\S 4$. Оболочки голоморфности вещественных поверхностей в комплексных поверхностях

иски в $\mathbb{C}^{2}$

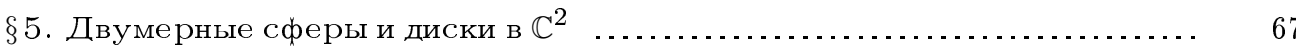

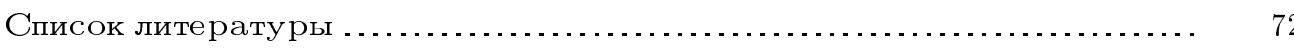

Любая голоморфная функция на компактном комплексном многообразии постоянна. Имеются ли какие-либо еше топологические препятствия для существования непостоянных голоморфных функций?

На одномерных комплексных многообразиях, т.е. римановых поверхностях, никаких других препятствий нет. Более того, как показали Бенке и Штейн, любая некомпактная риманова поверхность является многообразием Штейна, иначе говоря, допускает собственное голоморфное вложение в пространство $\mathbb{C}^{N}$. 
В то же время, в комплексной размерности 2 возникает ряд нетривиальных ограничений на поведение голоморфных функций, обусловленных гладкой топологией многообразия и, в частности, свойствами его двумерных вешественных подмногообразий вложенных вещественных поверхностей. В изучении этих вопросов важную роль сьграла следующая гипотеза, высказанная А. Г. Витушкиным в конце 80-х годов.

ГИПотеЗА ВитуШкина. Рассмотрим гладко вложсенную двумерную сферу $S$ в комплексной проективной плоскости $\mathbb{C P}^{2}$. Предположсим, ито сфера $S$ не гомологична нулю. Тогда любая голоморфная функиия в окрестности этой сферь постоянна.

Настоящая работа представляет собой обзор результатов и методов, необходимых для доказательства этой гипотезы и родственных ей утверждений о поведении голоморфных функций в окрестности вещественных поверхностей в комплексных поверхностях. Ключевым моментом является применение инвариантов Зайберга-Виттена гладких четырехмерных многообразий и в особенности результатов, полученных при доказательстве гипотезы Р. Тома о свойстве комплексных кривых минимизировать род в своем классе гомологий.

Можно рассматривать предлагаемьй обзор как "аналитическое продолжение" доклада [1], в котором описьвались топологические приложения возникшей в многомерном комплексном анализе техники подклеивания семейств голоморфных дисков. Мы же применим топологию для решения аналитических задач.

\section{$\S$ 1. Оболочки голоморфности и проблема Леви}

Одной из основных отличительных особенностей многомерного комплексного анализа является “принудительное" продолжение аналитических функций. Иначе говоря, в некоторых случаях все голоморфные функции в данной области на комплексном многообразии продолжаются в некоторую большую область. Разумеется, такое продолжение может приводить к многозначным аналитическим функциям, и потому получаюшаяся "оболочка голоморфности” оказывается областью над исходным многообразием. Основной вопрос состоит в геометрическом описании оболочек голоморфности.

В этом параграфе собраны необходимые в дальнейшем сведения об оболочках голоморфности, их свойствах и обобщениях.

1.1. Определение оболочек. Пусть $X$ - комплексное многообразие размерности $n$. Областью над $X$ называется связное комплексное многообразие $U$ вместе с локально биголоморфным отображением $p: U \rightarrow X$. Говорят, что область $(U, p)$ содержит область $(V, q)$, если задано отображение $j: V \rightarrow U$, согласованное с проекциями $p \circ j=q \circ j$. Отметим, что отображение $j$ может не быть инъективным. Однако, если область $(V, q)$ однолистна, т.е. отображение $q$ является вложением, то многообразие $V$ действительно является областью в $U$.

Оболочкой голоморфности $(\widetilde{U}, \widetilde{p})$ области $(U, p)$ над $X$ назьвается максимальная область над $X$, содержашая $(U, p)$ и такая, что любая голоморфная функция в $U$ голоморфно продолжается на $\widetilde{U}$. 
Нетрудно показать, что любая область $p: U \rightarrow X$ имеет единственную (с точностью до естественного изоморфизма) оболочку голоморфности над $X$. Элегантная конструкция оболочки с помошью пучков голоморфных функций приведена в книге [28]. Снова отметим, что только однолистная область заведомо содержится в своей оболочке в обычном смысле.

1.2. Псевдовыпуклость. Оболочка голоморфности области в каком-то смысле "лучше" самой области. В частности, она обладает некоторыми свойствами вьпуклости.

Исходньмм пунктом здесь является классическая теорема Картана-Туллена: оболочка голоморфности любой области над $\mathbb{C}^{n}$ является штейновым многообразием над $\mathbb{C}^{n}$. Оболочки голоморфности над общими многообразиями обладают аналогичными локальными свойствами. Область $p: U \rightarrow X$ называется псевдовыпуклой (или локально псевдовыпуклой), если у каждой точки $x \in X$ найдется окрестность $V \ni x$, прообраз которой $p^{-1}(V) \subset U$ является (возможно несвязнымм) многообразием Штейна. Из теоремы Картана-Туллена следует, что оболочка голоморфности любой области локально псевдовыпукла.

1.3. Проблема Леви. Локально псевдовыпуклые области совершенно не обязательно штейновы. Для данного комплексного многообразия $X$ проблема Леви состоит в установлении условий, при которых псевдовьпуклая область над $X$ (например, оболочка голоморфности некоторой области) является многообразием Штейна.

Для областей над $\mathbb{C}^{n}$ ответ дается теоремой Ока: любая локально псевдовыпуклая область над $\mathbb{C}^{n}$ штейнова. Заметим, что для оболочек голоморфности это утверждение эквивалентно теореме Картана-Туллена. Ниже мы кратко обсудим доказательство теоремы Ока.

Решение проблемы Леви над $\mathbb{C}^{n}$ непосредственно обобщается на многообразия Штейна. Вкладывая многообразие Штейна в $\mathbb{C}^{n}$ и используя ретракцию его штейновой окрестности, можно показать, что любая псевдовыпуклая область над многообразием ШІтейна также является многообразием Штейна.

1.4. Оболочки над проективными пространствами. В настояшее время проблема Леви решена только для некоторых классов комплексных многообразий. Случай комплексных проективных пространств и их прямых произведений был впервые рассмотрен Фуджита [13], [14]. Другие доказательства были даны Такеучи [41] и Чисельманом [20]. Мы следуем подходу Уэда [43], который обобшил этот результат на грассманианы.

Рассмотрим проективное пространство $\mathbb{C P}^{n}$ как фактор $\mathbb{C}^{n+1} \backslash\{0\}$ по действию мультипликативной группы $\mathbb{C}^{*}$. Голоморфные функции в области над $\mathbb{C P}^{n}$ соответствуют голоморфным функциям над $\mathbb{C}^{n+1}$, постоянным на комплексных прямых, проходящих через начало координат. Это свойство функций сохраняется при аналитическом продолжении в $\mathbb{C}^{n+1}$, поскольку его можно выразить дифференциальным уравнением

$$
\sum_{j=1}^{n+1} z_{j} \frac{\partial}{\partial z_{j}} f=0 .
$$


Таким образом, оболочки над $\mathbb{C P}^{n}$ соответствуют оболочкам над $\mathbb{C}^{n+1}$.

Теперь рассмотрим два случая. Если оболочка над $\mathbb{C}^{n+1}$ содержит начало координат, то все инвариантные функции на ней постоянны и оболочка над $\mathbb{C P}^{n}$ совпадает со всем пространством. В противном случае оболочка над $\mathbb{C}^{n+1}$ представляет собой многообразие Штейна, являющееся пространством главного расслоения с редуктивной структурной группой $\mathbb{C}^{*}$. Используя инвариантное интегрирование по максимальной компактной подгруппе, можно показать (см. [29]), что база этого расслоения, т.е. оболочка над $\mathbb{C P}^{n}$, также штейнова.

Итак, оболочка голоморфности области над $\mathbb{C P}^{n}$ либо является многообразием Штейна, либо совпадает со всем $\mathbb{C P}^{n}$. Иначе говоря, оболочка голоморфности штейнова тогда и только тогда, когда в области есть непостоянные голоморфные функции. Комбинируя это рассуждение с результатами предыдущего пункта, получаем следуюшую теорему.

Tеорема 1. Пусть $X=Y \times \mathbb{C P}^{n_{1}} \times \cdots \times \mathbb{C P}^{n_{k}}-$ прямое произведение штейнова многообразия $Y$ и комплексных проективных пространств. Тогда оболочка голоморфности любой области над $X$ либо является многообразием Штейна, либо содержит один из сомножителей $\mathbb{C P}^{n_{j}}$.

1.5. Плюрисубгармоничность и проблема Леви. Напомним, что гладкая вешественная функция $\psi$ на комплексном многообразии плюрисубгармонична (PSH), если ее форма Леви $L(\psi)=i \partial \bar{\partial} \psi$ является неотрицательной $(1,1)$-формой, иначеговоря, если комплексньй гессиан $H(\psi)=\left(\frac{\partial^{2} \psi}{\partial z_{j} \partial \bar{z}_{k}}\right)_{1 \leqslant j, k \leqslant n}$ является неотрицательной эрмитовой формой. Функция $\psi$ назьвается строго плюрисубгармонической (SPSH), если эти формы положительны. Аналогичным образом можно определить не обязательно гладкие PSH функции в терминах положительных $(1,1)$-потоков.

Относительно компактная область $U \subset X$ с гладкой границей на комплексном многообразии назьвается строго псевдовыпуклой, если в окрестности каждой граничной точки она имеет вид $\{\psi<0\}$ для гладкой SPSH функции $\psi$. Нетрудно видеть, что строго псевдовыпуклая область псевдовыпукла.

Проблема Леви для строго псевдовыпуклых областей существенно проще, чем в общем случае. А именно, Грауэрт [16] доказал, что строго псевдовыпуклая область итейнова тогда и только тогда, когда она не содержит компактных аналитических подмножеств.

Исходя из теоремы Грауэрта, можно следуюшим образом получить решение Ока проблемы Леви над $\mathbb{C}^{n}$. Основной факт состоит в том, что для локально псевдовыпуклой области $U \subset \mathbb{C}^{n}$ функция - $\log \operatorname{dist} \partial U(z)$ плюрисубгармонична (но только непрерьвна). Используя регуляризацию и добавляя стандартную SPSH функцию $\sum\left|z_{j}\right|^{2}$ в $\mathbb{C}^{n}$, получаем исчерпание области $U$ строго псевдовыпуклыми областями, к которым применима теорема Грауэрта.

Эта схема была обобшена Такеучи [42]. Он заметил, что для псевдовыпуклой области над кэлеровым многообразием форма Леви функции - $\log \operatorname{dist} \partial U(z)$ ограничена снизу на компактных множествах. Отсюда следует обобщение теоремы Ока на кэле- 
ровы многообразия при условии сушествования “фоновой” SPSH функции. Мы сформулируем несколько упрощенньй вариант теоремы Такеучи.

Теорема 2. Пусть $U \subset X$ - относительно компактная псевдовыпуклая область в кәлеровом многообразии. Предположим, ито в окрестности $\bar{U}$ существует строго плюрисубгармоническая функиия. Тогда область $U$ итейнова.

Этот результат можно использовать, например, для решения проблемы Леви над раздутиями штейновых многообразий. Заметим для этого, что если область $U$ пересекает исключительньй дивизор раздутия по строго псевдовьпуклой области, то нетрудно построить требуемую SPSH функцию на $\bar{U}$.

1.6. Принцип непрерьвности. Рассмотренные до сих пор результаты описывают свойства оболочек голоморфности, но фактически не дают методов построения оболочки голоморфности заданной области. Принцип непрерьвности показывает, что голоморфные функции продолжаются вдоль непрерьвных семейств аналитических дисков. Мы сформулируем только простой локальный вариант (по поводу обобщений см. [6] и $[18])$.

Предположим, что для области $U \subset \mathbb{C}^{n}$ существует непрерывное семейство вложенных аналитических дисков $f_{t}: \bar{D} \rightarrow \bar{U}, t \in[0,1]$, со следующими свойствами:

(1) $f_{t}(\bar{D}) \subset U$ npu $t<1$

(2) $f_{t}(\partial D) \subset K$ для всех $t \in[0,1]$ и фиксированного компакта $K \subset U$;

(3) $f_{1}(D) \cap \partial U=\{p\}$ для некоторой точки $p \in \partial U$.

Тогда любая голоморфная в области $U$ функиия продолжается в точку $p$.

Этот результат можно применить, в частности, к классическому приспособлению для аналитического продолжения - фигуре Хартогса.

Рассмотрим множество $H=\{|z|=1,|w| \leqslant 1\} \cup\{w=0,|z| \leqslant 1\} \subset \mathbb{C}^{2}$. Это объединение единичного диска в плоскости $z$ с “боковыми по $z$ ” стенками единичного бидиска. Применяя принцип непрерывности к семействам дисков $f_{t}(z)=(z, t) \in \mathbb{C}^{2}$, $t \in \mathbb{C}$, мы видим, что любая голоморфная функция в окрестности $H$ голоморфно продолжается в окрестность всего бидиска.

\section{§2. Комплексные точки и штейновы окрестности вешественньх поверхностей в комплексньх поверхностях}

В этом параграфе изложен метод построения штейновых окрестностей подходяшим образом выбранных вещественных поверхностей. Мы постарались дать по возможности элементарное и замкнутое изложение необходимых результатов Лая, Харламова-Элиашберга и других авторов об изотопиях вложенных вещественных поверхностей в комплексных поверхностях.

Основное утверждение состоит в том, что вложенная вещественная поверхность, топологические характеристики которой удовлетворяют так назьваемым неравенствам присоединения, изотопна поверхности с базисом штейновых окрестностей. Эта теорема затем используется для построения примеров штейновых областей с интерес- 
ными геометрическими свойствами. В дальнейшем с ее помошью мы убедимся в точности получаемых из теории Зайберга-Виттена оценок для рода вешественных поверхностей в штейновых поверхностях.

2.1. Комплексные точки вещественных поверхностей в комплексных поверхностях. Пусть $S \subset X$ - вешественная поверхность, вложенная в комплексную поверхность. Точка $p \in S$ назьвается комплексной (или $\mathbb{R} \mathbb{C}$-особой), если касательная плоскость $T_{p} S \subset T_{p} X$ является комплексной прямой.

Локально вешественная поверхность $S$ задается графиком гладкой комплекснозначной функции $f$, и точка $(z, f(z)) \in S$ является комплексной тогда и только тогда, когда $\frac{\partial f}{\partial \bar{z}}(z)=0$. Поэтому нетрудно показать, что для вложений общего положения комплексные точки изолированы и вблизи каждой комплексной точки найдется система комплексных координат $(z, w)$, в которой поверхность $S$ задается уравнением вида

$$
w=|z|^{2}+\frac{\gamma}{2}\left(z^{2}+\bar{z}^{2}\right)+\bar{o}\left(|z|^{2}\right), \quad \gamma \in \mathbb{R}
$$

Если $|\gamma|<1$, то комплексная точка $p=(0,0)$ назьвается эллиптической, а если $|\gamma|>1$, то әиперболической. ("Параболический" случай $|\gamma|=1$ не является случаем общего положения.) Таким образом, если поверхность $S$ есть график $\Gamma_{f}$, то эллиптические и гиперболические комплексные точки соответствуют положительным и отрицательным нулям производной $\frac{\partial f}{\partial \bar{z}}$.

Заметим, что свойство комплексной точки быть эллиптической или гиперболической инвариантно относительно биголоморфных замен координат.

2.2. Подсчет числа комплексных точек. Предположим, что вложенная вещественная поверхность $S \subset X$ в общем положении компактна и ориентирована. Тогда в каждой комплексной точке $p \in S$ имеются две ориентации касательной плоскости $T_{p} S$. Во-первых, ориентация поверхности $S$, а во-вторых, каноническая комплексная ориентация на прямых в $T_{p} X$. Комплексная точка положительна, если эти две ориентации совпадают, и отрицательна в противном случае.

Обозначим через $e_{ \pm}=e_{ \pm}(S)$ и $h_{ \pm}=h_{ \pm}(S)$ число положительных и отрицательных эллиптических и гиперболических точек поверхности $S$. Введем индексы Лая $I_{ \pm}:=e_{ \pm}-h_{ \pm}$. Оказывается, что числа $I_{ \pm}$являются топологическими инвариантами вложения.

ФормУЛЫ ЛАЯ [25]. Пусть $S \subset X$ - компактная ориентированная вещественная поверхность общего положения в комплексной поверхности X. Тогда

$$
\begin{aligned}
& I_{+}+I_{-}=\chi(S)+S^{2}, \\
& I_{+}-I_{-}=c_{1}(X) \cdot S,
\end{aligned}
$$

где $\chi(S)=2-2 g(S)$ - эйлерова характеристика, $S^{2}=[S] \cdot[S]-$ индекс самопересечения поверхности $S$ в $X$, а $c_{1}(X) \in H^{2}(X, \mathbb{R})$ - первый класс Черна многообразия $X$. 
ДокАЗАТЕльСтво. Приведем только набросок доказательства. Для вывода первой формулы выберем касательное векторное поле $\xi \in T S$, применим к нему оператор $J$ комплексной структуры на $X$ и рассмотрим проекцию $\eta=\pi(J \xi) \in N S$ на нормальное расслоение $S$. Нули $\eta$ суть в точности нули $\xi$ и комплексные точки $S$. Аккуратное вычисление знаков (с помошью уравнений $(*))$ дает первую формулу Лая.

Для доказательства второй формулы зафиксируем не обрашаюшуюся в нуль 2-форму $\omega$ на $S$ и заметим, что для любого локального репера $\xi_{1}, \xi_{2} \in T S$ выражение $\omega\left(\xi_{1}, \xi_{2}\right)^{-1} \xi_{1} \wedge_{\mathbb{C}} \xi_{2}$ определяет сечение линейного расслоения $\left.\Lambda_{\mathbb{C}}^{2}(T X)\right|_{S}$. Нули этого сечения суть комплексные точки $S$, поскольку именно в этих точках поля $\xi_{1}$ и $\xi_{2}$ линейно зависимы над $\mathbb{C}$. Остается найти знаки этих нулей и воспользоваться определением $c_{1}(X)$.

Иногда удобно записьвать формулы Лая в виде

$$
2 I_{ \pm}(S)=\chi(S)+S^{2} \pm c_{1}(X) \cdot S
$$

Например, если $C \subset X-$ компактная комплексная кривая, то после малого вешественного шевеления мы получим вложенную вешественную поверхность $S$, которая имеет только положительные комплексные точки. Таким образом, $I_{-}(S)=0$ и, следовательно, $-\chi(C)=C^{2}-c_{1}(X) \cdot C$, что совпадает с формулой присоединения для кривой $C$.

Можно дать также следуюшую геометрическую интерпретацию индексов Лая. Рассмотрим грассманово многообразие $\operatorname{Gr}(X, \mathbb{R}, 2)$ ориентированных вещественных двумерных плоскостей в касательном расслоении $T X$. В нем имеются два естественных ориентированных подмногообразия $\mathfrak{I}_{ \pm}$, образованных комплексными прямыми с комплексной и анти-комплексной ориентацией. Тогда $I_{ \pm}=\tau(S) \cdot \mathfrak{I}_{ \pm}$, где $\tau: S \rightarrow G r(X, \mathbb{R}, 2)$ - касательное гауссово отображение. Это утверждение также следует из вычисления локальных индексов.

2.3. Сокрашение комплексных точек. Индексы Лая $I_{ \pm}$являются по сушеству единственными топологическими инвариантами расположения вложенных вещественных поверхностей относительно комплексной структуры. Следуюшая теорема Харламова и Элиашберга показьвает, как можно устранить эллиптическую и гиперболическую точки одного знака. В частности, вложенная поверхность с $I_{+}=I_{-}=0$ изотопна вполне вещественной поверхности. Это утверждение представляет собой частньй случай $h$-принципа Громова для $C R$-вложений.

Теорема 3 (о сокрашении). Пусть $S \subset X-$ вложенная ориентированная вещественная поверхность в общем положении. Eсли е $e_{+}(S) h_{+}(S)>0$, то существует изотопная вложенная вещественная поверхность $S^{\prime} \subset X$, для котоpoй $e_{+}\left(S^{\prime}\right)=e_{+}(S)-1$ u $h_{+}\left(S^{\prime}\right)=h_{+}(S)-1$.

Соединим рассматриваемую пару комплексных точек гладким сохраняющим ориентацию путем $\gamma$ на поверхности $S$, не проходяшим через другие комплексные точки. Окрестность $U \supset \gamma$ можно так биголоморфно отобразить в $\mathbb{C}^{2}$, что часть поверхности $S \cap U$ перейдет в график комплексной функции. Для этого нужно сначала выбрать 
диффеоморфизм, обладающий этим свойством и такой, что его дифференциал комплексно-линеен в точках $\gamma$, а затем приблизить его голоморфными отображениями в штейновых окрестностях вешественной кривой $\gamma$ (детали см. в [12; с. 370]). Это рассуждение сводит теорему к следующему локальному результату.

Лемма 1. Пусть $f: \bar{\Delta} \rightarrow \mathbb{C}$ - гладкая комплексная функция в окрестности единичного диска. Предположсим, что производная $\frac{\partial f}{\partial \bar{z}}$ имеет два простых нуля $a, b \in \Delta$ противоположсного знака. Тогда существует гладкая функиия $g: \bar{\Delta} \rightarrow \mathbb{C}$, близкая $\kappa f$ в $C^{0}$-норме на диске, совпадающая $c f$ вблизи границь диска и такая, что $\frac{\partial g}{\partial \bar{z}}$ нигде не обращается в нуль.

ДокАЗАТЕльство. Основа доказательства - это следующее свойство $\bar{\partial}$-уравнения. Пусть $\psi$ - гладкая функция с носителем в $\Delta$, тогда формула Коши

$$
u(z)=\frac{1}{2 \pi i} \int \frac{\psi(\zeta+z)}{\zeta} d \zeta \wedge d \bar{\zeta}
$$

задает решение уравнения $\frac{\partial u}{\partial \bar{z}}=\psi$, для которого $\|u\|_{C^{0}} \leqslant$ const $\cdot\|\psi\|_{L^{p}}, p>2$. Эта оценка следует из неравенства Гёльдера, поскольку ядро Коши $\frac{1}{\zeta}$ лежит в $L_{\mathrm{loc}}^{q}$ для любого $q<2$.

Теперь мы можем доказать лемму. По очевидньм топологическим причинам существует гладкая функция $\psi$ с носителем в малой окрестности отрезка $[a, b]$, для которой сумма $\frac{\partial f}{\partial \bar{z}}+\psi$ не обрашается в нуль нигде в $\Delta$. Заметим, что функцию $\psi$ можно выбрать так, что $\|\psi\|_{C^{0}} \leqslant 2\left\|\frac{\partial f}{\partial \bar{z}}\right\|_{C^{0}}$. Следовательно, если плошадь носителя $\operatorname{supp}(\psi)$ достаточно мала, то соответствуюшая функция $u$ имеет малую $C^{0}$-норму, а график функции $f+u$ вполне вешественен. Остается домножить функцию $u$ на отсекаюшую функцию, чтобы получить $u \equiv 0$ вблизи границы $\Delta$. Эта операция "не испортит" $\frac{\partial(f+u)}{\partial \bar{z}}$, поскольку функция $u$ голоморфна вблизи границы и имеет малую $C^{0}$-норму.

2.4. Окрестности вещественных поверхностей. Теперь можно построить штейновы окрестности деформаций вешественных поверхностей, удовлетворяющих подходяшим топологическим условиям.

Заметим сразу, что вложенная вешественная поверхность $S \subset X$ с эллиптическими комплексными точками не может иметь базиса штейновых окрестностей. Дело в том, что согласно теореме Бишопа [2] для эллиптической комплексной точки $p \in S$ существует нетривиальное непрерывное семейство голоморфных дисков $f_{t}:(\Delta, \partial \Delta) \rightarrow$ $(X, S)$, для которого $f_{0} \equiv p$. По принципу непрерывности любая голоморфная функция в окрестности $S$ голоморфно продолжается вдоль этого семейства, и потому никакая достаточно малая окрестность $S$ не может быть штейновой.

Условие следуюшей теоремы как раз и состоит в возможности устранить эллиптические комплексные точки с помощью изотопии. 
ТЕОРемА 4. Пусть $S \subset X$ - компактная ориентированная вложенная вещественная поверхность в комплексной поверхности. Если $S$ удовлетворяет обоим неравенствам

$$
2 I_{ \pm}=\chi(S)+S^{2} \pm c_{1}(X) \cdot S \leqslant 0
$$

то существует изотопная вложсенная вещественная поверхность с фундаментальной системой штейновых окрестностей.

Этот результат был доказан в [12] при $X=\mathbb{C}^{2}$. Случай, когда $S$ является вещественной деформацией комплексной кривой, использовался в [33], а обший случай в [34].

ДокАЗАТЕльство. По теореме о сокрашении существует изотопная $S$ вешественная поверхность, которую мы снова обозначим через $S$, имеющая только гиперболические комплексные точки.

Рассмотрим вполне вешественную илигиперболическую комплексную точку $p \in S$. Из локального уравнения в п. 2.1 для $S$ нетрудно видеть, что сушествуют координатная окрестность $U \ni V \ni p$ и неотрицательная гладкая функция $\psi$ на $U$ со следуюшими свойствами:

1) $S \cap U=\{x \in U \mid \psi(x)=0\}$;

2) $d \psi \equiv 0$ на $S \cap U$;

3) $\psi$ строго плюрисубгармонична на $U \backslash(S \cap V)$.

Выберем конечное покрытие $S$ окрестностями $V_{j} \Subset U_{j}$. Пусть, кроме того, $\chi_{j}-$ такие гладкие неотрицательные функции с носителями в $U_{j}$, что $\chi_{j} \equiv 1$ на $\bar{V}_{j}$ и $\sum_{|\alpha| \leqslant 2}\left|D^{\alpha} \chi_{j}\right| \leqslant A \chi_{j}^{1 / 3}$ всюду в $U_{j}$ для некоторой константы $A$. Такие функции существуют: можно сначала выбрать функции, удовлетворяющие первому условию, а затем возвести их в куб.

Из формулы Лейбница следует, что форма Леви $L\left(\chi_{j} \psi_{j}\right)$ неотрицательна в некоторой окрестности $W_{j} \supset S \cap U_{j}$ и положительна в $\left(W_{j} \backslash S\right) \cap V_{j}$.

Рассмотрим функцию $\Phi:=\sum_{j} \chi_{j} \psi_{j}$. По построению

1) $\Phi \equiv 0$ на $S$ и $\Phi>0$ вне $S$;

2) $\Phi$ строго плюрисубгармонична в проколотой окрестности поверхности $S$.

Итак, множества $U_{\varepsilon}=\{\Phi<\varepsilon\}$, где $\varepsilon>0$ - регулярное значение функции $\Phi$, образуют базис строго псевдовыпуклых штейновых окрестностей поверхности $S$.

В $\S 3$ мы воспользуемся инвариантами Зайберга-Виттена и покажем, что если вещественная поверхность $S$ “потенциально" имеет эллиптические комплексные точки (т.е. один из индексов $I_{ \pm}(S)$ положителен), то она не может лежать в (не обязательно малой) штейновой области, за исключением случая гомологичной нулю двумерной сфферы.

2.5. Приложения. Простейшие применения наших результатов относятся к вложенным вешественным поверхностям в $\mathbb{C}^{2}$. Поскольку $H^{2}\left(\mathbb{C}^{2}, \mathbb{Z}\right)=0$, по формулам Лая $I_{ \pm}(S)=1-g(S)$. Таким образом, любая вложенная ориентированная поверхность положительного рода в $\mathbb{C}^{2}$ изотопна поверхности без эллиптических точек 
и с базисом штейновых окрестностей. В то же время, по теореме 15 любая вложенная 2-сфера $S \subset \mathbb{C}^{2}$ гомологична нулю в своей оболочке голоморфности, и, следовательно, любая штейнова окрестность $S$ должна быть достаточно "велика" (см. также [35]).

Теорему 4 можно также использовать для построения штейновых областей с интересными топологическими свойствами.

Рассмотрим компактную комплексную алгебраическую поверхность $X$ и неособое гиперплоское сечение $C \subset X$, т.е. комплексную кривую, пересекающую все кривые на $X$. Возможны два случая. Если $c_{1}(X) \cdot C \leqslant 0$, то общая вложенная вещественная поверхность $S$, изотопная комплексной кривой $C$, удовлетворяет неравенствам $I_{ \pm}(S) \leqslant 0$ в силу формулы присоединения и формул Лая. Если же $c_{1}(X) \cdot C>0$, то нужно сначала добавить $c_{1}(X) \cdot C$ тривиальных ручек к $C$, а затем получить поверхность $S$ с неотрищательными индексами Лая общим шевелением. Так или иначе, в обоих случаях изотопией $S$ мы получаем вложенную вешественную поверхность $S^{\prime}$, гомологичную комплексной кривой $C$ и имеюшую базис штейновых окрестностей. Такие штейновы области пересекают все комплексные кривые в $X$, поскольку для любой кривой $L$ индекс пересечения $L \cdot S^{\prime}=L \cdot C>0$.

Пусть теперь $Y$ - проективное многообразие размерности $n \geqslant 3$. Выберем $n-1$ гиперплоское сечение $H_{j} \subset Y$ в общем положении и рассмотрим поверхность $X=$ $\bigcap_{j=1}^{n-2} H_{j}$ и ее гиперплоское сечение $C=\bigcap_{j=1}^{n-1} H_{j}$. Пусть $V \subset X$ - построенная выше штейнова окрестность вешественной поверхности, гомологичной кривой $C$. По теореме Сью [38] о штейновых окрестностях штейновых подмногообразий сушествует штейнова область $U \subset Y$, для которой $U \cap X=V$. Мы заключаем, что имеет место следующий результат [34].

Теорема 5. Пусть $X$ - проективное многообразие размерности $n \geqslant 2$. Тогда существует такая строго псевдовыпуклая штейнова область $U \subset X$, что для любой комплексной гиперповерхности $H \subset X$ двойственный класс когомологий $[H] \in H^{2}(U, \mathbb{R})$ отличен от нуля. В частности, $U$ пересекает все гиперповерхности и потому не лежит ни в какой аффинной части многообразия $X$.

Поясним эту теорему следуюшими двумя примерами. Пусть $X=\mathbb{C P}^{2}$, и пусть $C-$ проективная прямая. Поскольку $c_{1}\left(\mathbb{C P}^{2}\right) \cdot C=3$, существует вложенная поверхность рода 3 , гомологичная проективной прямой и имеющая базис штейновых окрестностей.

ЗАМЕЧАнИЕ. В п. 4.1 мы покажем, что три - это действительно минимальный возможный род для такого примера. Заметим еще, что согласно результатам Бора [4] вложенная вешественная поверхность рода 3 и степени -1 в $\mathbb{C P}^{2}$ псевдоголоморфна относительно подходящей почти комплексной структуры $J$, изотопной стандартной интегрируемой структуре. Однако эта структура не подчинена симплектической форме Фубини-Штуди (ср. п. 4.5).

Пусть теперь $X=\mathbb{C P}^{3}$, и пусть снова $C$ - это проективная прямая. Существует гладкая комплексная гиперповерхность $H$ степени 4 , содержащая $C$. По формуле присоединения $c_{1}(H)=0$, и, следовательно, кривая $C \subset H$ изотопна вполне вешественной сфере. Таким образом, мы получаем штейнову область в $\mathbb{C P}^{3}$, содержащую гладкую вешественную деформацию проективной прямой. 


\section{§3. Инварианты Зайберга-Виттена и неравенства для рода вложенных поверхностей}

В этом параграфе изучается связь между комплексным анализом и топологией гладких четырехмерных многообразий, подсказанная результатами $§ 2$.

Сначала мы обсуждаем свойства инвариантов Зайберга-Виттена и выводим оценки для рода вешественных поверхностей в компактных кэлеровых поверхностях, а затем переносим эти результаты на штейновы поверхности с помошью алгебраической аппроксимации многообразий Штейна.

3.1. Сигнатура четырехмерных многообразий и комплексных поверхностей. Рассмотрим компактное ориентированное многообразие $X$ вешественной размерности 4. Форма пересечения на его двумерных когомологиях $H^{2}(X, \mathbb{R})$ симметрична и потому определены ее индексы инерции $b^{ \pm}(X)$. По определению сигнатурой многообразия $X$ называют сигнатуру $\sigma(X)=b^{+}-b^{-}$его формы пересечения.

Если $X$ является подлежашим гладким многообразием кэлеровой комплексной поверхности, то максимальное положительное подпространство в $H^{2}(X, \mathbb{R})$ можно описать следуюшим образом. Оно порождается классом кэлеровой формы $\omega$ и классами вешественных и мнимых частей голоморфных 2 -форм на $X$. В частности, $b^{+}(X)=$ $1+2 \operatorname{dim}_{\mathbb{C}} \Omega^{2}(X)$ - нечетное положительное число.

3.2. Инвариант Зайберга-Виттена. Пусть теперь $X$ - это компактное ориентированное гладкое четырехмерное многообразие с $b^{+}(X)>1$. Выберем характеристический класс когомологий $c \in H^{2}(X, \mathbb{Z})$, т.е. целочисленньй класс, сравнимый $\bmod 2$ со вторым классом Штифеля-Уитни $w_{2}(X) \in H^{2}\left(X, \mathbb{Z}_{2}\right)$ многообразия $X$. (Например, первьй класс Черна любой почти комплексной структуры $J$ на многообразии $X$ является характеристическим.)

В этой ситуации определен инвариант Зайберга-Виттена $S W(X, c) \in \mathbb{Z} / 2 \mathbb{Z}$ гладкого ориентированного многообразия $X$. Это означает, что если $\psi$ - сохраняюший ориентацию диффеоморфизм многообразия $X$, то $S W\left(X, \psi^{*} c\right)=S W(X, c)$.

Грубо говоря, инвариант $S W(X, c)$ отличен от нуля, если многообразие модулей решений дифференциальных уравнений Зайберга-Виттена для римановой метрики на $X$ не гомологично нулю в конфигурационном пространстве. Точное определение инварианта и доказательство используемых ниже свойств можно найти в оригинальной работе Виттена [44] и в монографииях [31] и [30].

Инвариант Зайберга-Виттена можно определить также при $b^{+}=1$, но в этом случае он зависит от дополнительных параметров (см., например, [22] и [36]).

3.3. Инварианты Зайберга-Виттена компактных комплексных поверхностей. Предположим теперь, что $X$ является компактной кэлеровой поверхностью. Тогда, как указал Виттен, пространство модулей решений (подходящим образом возмушенных) уравнений Зайберга-Виттена для кэлеровой метрики может быть отождествлено с пространством всех эффективных дивизоров, двойственных по Пуанкаре классу двумерных когомологий $\frac{1}{2}\left(c-c_{1}(X)\right)$.

В частности, на кэлеровой поверхности с $b^{+}(X)>1$ инвариант Зайберга-Виттена $S W\left(X, c_{1}(X)\right)=1$, поскольку в этом случае пространство модулей состоит из единственной точки, соответствующей нулевому дивизору. 
В ином направлении можно использовать инварианты Зайберга-Виттена для нахождения комплексных кривых с заданным классом когомологий на $X$ : если $S W(X, c) \neq 0$, то сушествует (возможно приводимая) комплексная кривая, двойственная по Пуанкаре классу $\frac{1}{2}\left(c-c_{1}(X)\right)$.

3.4. Оценки решений. Пусть инвариант $S W(X, c)$ многообразия $X$ отличен от нуля. Тогда, практически по определению, уравнения Зайберга-Виттена для любой метрики $g$ имеют решение. Используя свойства уравнений, удается получить априорные оценки решений в терминах метрики $g$. Мы не будем выписывать сами уравнения, а приведем ключевую оценку такого типа, принадлежашую самому Виттену (см. также [30], [21]).

Пусть $g$ - риманова метрика на $X$ со скалярной кривизной $s_{g}$. Если инвариант $S W(X, c) \neq 0$ для некоторого класса $c \in H^{2}(X, \mathbb{Z})$, то сушествует замкнутая 2-форма $\eta$, представляющая этот класс в когомологиях де Рама и такая, что

$$
\|\eta\|_{L_{g}^{2}} \leqslant \frac{1}{4 \pi}\left\|s_{g}\right\|_{L_{g}^{2}}+K(X)
$$

где константа $K(X)$ зависит только от топологии многообразия $X$.

В качестве следствия мы получаем, что число классов $c \in H^{2}(X, \mathbb{Z})$ с нетривиальным инвариантом $S W(X, c)$ конечно. Действительно, для данной метрики $g$ гармонические представители этих целочисленных классов равномерно ограничены по норме.

3.5. Простейшее неравенство для рода. Пусть $\Sigma \subset X$ - вложенная ориентированная вешественная поверхность в компактном ориентированном четырехмерном многообразии $X$, для которого $b^{+}>1$. Предположим, что индекс самопересечения $\Sigma^{2}=0$ и, следовательно, окрестность поверхности $\Sigma$ диффеоморфна прямому произведению $\Sigma \times D^{2}$. Следуюший результат был получен Кронхеймером и Мрувкой [22]. Мы используем подход Кронхеймера [21].

ПрЕДЛОЖЕНИЕ. Ecли $S W(X, c) \neq 0, \operatorname{mo}|c \cdot \Sigma| \leqslant \max \{0,-\chi(\Sigma)\}$.

Таким образом, любая гладко вложенная вешественная поверхность, реализующая класс гомологий с ненулевым пересечением с классом $c \in H^{2}(X, \mathbb{Z})$, должна иметь достаточно большой род, быть “достаточно сложной”.

ДокАЗАтЕльство. Заметим сразу, что можно заменить поверхность $\Sigma$ гомологичной поверхностью большего рода и считать, что $\chi(\Sigma)=2-2 g(\Sigma) \leqslant 0$.

Введем семейство римановых метрик $g_{R}, R>0$, на многообразии $X$ таким образом, что в трубчатой окрестности поверхности $\Sigma$ метрика $g_{R}$ совпадает с прямой суммой метрики постоянной скалярной кривизны $4 \pi \chi(S)$ на $\Sigma$ и плоской метрики $R^{2}\left(d x^{2}+d y^{2}\right)$ на диске $D^{2}$. Пусть, кроме того, метрики $g_{R}$ не зависят от $R$ вне некоторой большей окрестности $\Sigma$. В соответствии с формулой Гаусса-Бонне мы можем считать, что площадь поверхности $\Sigma$ в любой из этих метрик равна 1.

Пусть теперь $\omega_{R}$ - гармонический представитель класса $c$ относительно метрики $g_{R}$. Поскольку гармоническая форма минимизирует $L^{2}$-норму в своем классе когомологий, из оценки п. 3.4 следует неравенство

$$
\left\|\omega_{R}\right\|_{L_{g_{R}}^{2}} \leqslant-\chi(\Sigma) \sqrt{\pi} R+O(1), \quad R \rightarrow \infty .
$$


$\mathrm{C}$ другой стороны, поскольку $\omega_{R}$ представляет $c \cdot \Sigma$ в $H^{2}(\Sigma, \mathbb{R}) \cong \mathbb{R}$, мы получаем

$$
\left\|\omega_{R}\right\|_{L_{g_{R}}^{2}} \geqslant|c \cdot \Sigma| \sqrt{\pi} R
$$

Эти два асимптотических неравенства совместны тогда и только тогда, когда

$$
-\chi(\Sigma) \geqslant|c \cdot \Sigma|
$$

что и требовалось доказать.

3.6. Неравенство для рода поверхностей с неотрицательным индексом самопересечения. Пусть $X$ - компактная кэлерова поверхность с $b^{+}>1$, и пусть $S \subset X$ - вложенная вещественная поверхность с неотрицательным индексом самопересечения $S^{2} \geqslant 0$. Выберем ориентацию на $S$ так, что $c_{1}(X) \cdot S \leqslant 0$.

Раздуем поверхность $X$ в $d=S^{2}$ различных точках и обозначим раздутую поверхность через $\widetilde{X}$, исключительные кривые раздутий через $E_{j}$, а соответствующее стягивание через $\pi: \widetilde{X} \rightarrow X$. Тогда $c_{1}(\widetilde{X})=\pi^{*} c_{1}(X)-\sum_{j}\left[E_{j}\right]$.

Пусть $\Sigma$-внутренняя связная сумма $S$ с поверхностями $\bar{E}_{j}$ в многообразии $\widetilde{X}$ ("собственньй прообраз $\Sigma$ при $\pi$ "). Тогда $[\Sigma]=\pi^{*}[S]-\sum_{j}\left[E_{j}\right]$ и род поверхности $\Sigma$ равен роду $S$.

По предложению п. 3.5 мы получаем

$$
\max \{0,-\chi(S)\} \geqslant\left|c_{1}(\tilde{X}) \cdot \Sigma\right|=\left|c_{1}(X) \cdot S-S^{2}\right|=\left|c_{1}(X) \cdot S\right|+S^{2} .
$$

Отсюда уже несложно вывести известный результат Кронхеймера-Мрувки [22] и Моргана-Сабо-Таубса [32].

Теорема 6. Вложсенная вещественная поверхность $S \subset X$ с неотрицательным индексом самопересечения в компактной кәлеровой поверхности с $b^{+}(X)>1$ удовлетворяет неравенству

$$
S^{2}+\left|c_{1}(X) \cdot S\right| \leqslant-\chi(S)
$$

если только $S$ - это не сфера с нулевым вещественным классом гомологий.

ДокАЗАТЕльство. Остается проверить, что вложенная сфера с $S^{2} \geqslant 0$ в кэлеровой поверхности с $b^{+}>1$ гомологична нулю над $\mathbb{R}$. По доказанному неравенству $S^{2}=0$ и $c_{1}(X) \cdot S=0$.

Раздуем поверхность $X$ в одной точке. Пусть $E \subset \widetilde{X}$ - исключительная кривая раздутия. Для произвольного целого $n \geqslant 1$ рассмотрим внутреннюю связную сумму $S_{n}=E \# n S$. Это вложенная сфера, для которой $S_{n}^{2}=-1$ и $c_{1}(\tilde{X}) \cdot S_{n}=1$.

Воспользуемся теперь тем, что сушествует сохраняюший ориентацию диффеоморфизм $\psi: \widetilde{X} \rightarrow \widetilde{X}$, тождественньй вне окрестности сферы $S_{n}$ и переводящий $S_{n}$ в себя с противоположной ориентацией. Имеем $\psi^{*} c_{1}(\widetilde{X})=c_{1}(\widetilde{X})+2\left[S_{n}\right]$. Если класс $[S] \in H^{2}(X, \mathbb{R})$ не нулевой, то получается бесконечное множество классов на $\widetilde{X}$ с ненулевым инвариантом Зайберга-Виттена, что противоречит п. 3.4. 
ЗАмЕчАниЕ. Согласно формуле для рода комплексная кривая $C$ с неотрицательным индексом самопересечения $C^{2} \geqslant 0$ не удовлетворяет нашему неравенству тогда и только тогда, когда $c_{1}(X) \cdot C>0$. Такая ситуация действительно возникает в $\mathbb{C P}^{2}$ и других поверхностях с отрищательным каноническим классом. Поэтому, в частности, в доказанной теореме нельзя опустить условие $b^{+}>1$.

В $\S 4$ мы не раз используем тот факт, что комплексные кривые в поверхностях с $c_{1}(X)>0$ не удовлетворяют неравенству для рода "общего" вида.

3.7. Поверхности с отрицательным самопересечением. Если индекс самопересечения вложенной поверхности отрицателен, то она может и не удовлетворять неравенству из теоремы 6. Имеет место несколько иное утверждение, вытекающее из результатов Финташела и Стерна [10] и Ожвата и Сабо [36]. Отметим, впрочем, что здесь уже не играет роли условие $b^{+}>1$.

ТЕОРема 7. Пусть $S \subset X-$ вложсенная ориентированная вещественная поверхность с отрицательным индексом самопересечения в компактной кәлеровой поверхности. Если $S^{2}+\left|c_{1}(X) \cdot S\right|>-\chi(S)$, то один из классов $\pm S$ представим (возможно, приводимой) комплексной кривой.

Для доказательства этой теоремы необходимо показать, что если поверхность $S$ не удовлетворяет естественному неравенству для рода, то для одного из классов $c_{1}(X) \pm 2[S]$ отличен от нуля инвариант Зайберга-Виттена (определенный с учетом случая $\left.b^{+}=1\right)$. Именно такая ситуация возникла для $(-1)$-сфер в п. 3.6. После этого нужно применить п. 3.3 для нахождения искомой комплексной кривой.

3.8. Обобщенная гипотеза Тома. Неравенства п. 3.5 и п. 3.6 были впервые получены Кронхеймером и Мрувкой [22] при доказательстве следующей топологической гипотезы, обычно приписываемой Рене Тому: неособая комплексная кривая на комплексной алгебраической поверхности имеет минимальный род в своем классе гомологий.

Род неособой кривой $C \subset X$ дается формулой присоединения

$$
C^{2}-c_{1}(X) \cdot C=2 g-2
$$

Поэтому, например, из теоремы 6 следует гипотеза Тома для случая $C^{2} \geqslant 0$ и $b^{+}(X)>1$.

В настоящее время гипотеза Тома полностью доказана и даже обобщена на симплектические кривые в симплектических многообразиях. Случай $C^{2} \geqslant 0$ был рассмотрен Морганом, Сабо и Таубсом [32], а случай $C^{2}<0$ - Ожватом и Сабо [36].

3.9. Алгебраическая аппроксимация многообразий Штейна. Сейчас мы оставим ненадолго инварианты Зайберга-Виттена, чтобы описать способ переноса полученных результатов на штейновы поверхности. На самом деле, можно развить всю теорию внутренним образом даже в более общем контексте "выпуклых симплектических многообразий” (см. [23]). Однако мы предпочитаем применить алгебраическую аппроксимацию и свести задачу к компактным алгебраическим поверхностям, как это было сделано в [27], [34] и [35]. 
Рассмотрим вложенное многообразие Штейна $Y \subset \mathbb{C}^{n}$. Для заданного компакта $K \subset Y$ мы хотим приблизить многообразие $Y$ аффинным алгебраическим многообразием $V$, так что окрестность компакта $K$ будет биголоморфна некоторому открытому множеству в $V$. Решение этой задачи получается с помошью следуюшего рассуждения, восходящего к Нэшу для вешественных многообразий и развитого Стаутом [40] и Демайи, Лемпертом и Шиффманом [7], [26].

Зафиксируем замкнутый полидиск $\Delta \supset K$. Сушествует конечный набор голоморфных функций $F_{1}, \ldots, F_{m}$, порождающих пучок идеалов подмногообразия $Y \subset \mathbb{C}^{n}$ в каждой точке $z \in \Delta$. Если $m=\operatorname{codim}_{\mathbb{C}} Y$, то мы можем просто приблизить функции $F_{1}, \ldots, F_{m}$ полиномами $P_{1}, \ldots, P_{m}$ и взять в качестве $V$ их множество нулей по теореме о неявной функции.

Однако в обшем случае необходимо учесть соотношения между $F_{1}, \ldots, F_{m}$. Для этого выберем голоморфные $\mathbb{C}^{m}$-значные функции $R_{1}, \ldots, R_{k}$, порождаюшие пучок соотношений $\mathscr{R}_{F_{1}, \ldots, F_{m}}$ в каждой точке $z \in \Delta$. Если одновременно приблизить голоморфные функции $F_{1}, \ldots, F_{m}$ и $R_{1}, \ldots, R_{k}$ полиномами с сохранением соотношений $\sum_{\ell=1}^{m} R_{j}^{\ell} F_{\ell}=0, j=1, \ldots, k$, то соотвествуюшее нулевое множество даст требуемое многообразие $V$.

Теорема 8. Пусть $K \subset Y$ - компактное множество в многообразии IIтейна. Тогда окрестность $К$ биголоморфно эквивалентна открытому множеству в аффинном алгебраическом многообразии.

ЗАмЕчАнИЕ. Если задано комплексное подмногообразие $Z \subset Y$, то с помошью такого же рассуждения можно добиться, чтобы пересечение $Z$ с окрестностью $K$ отображалось на пересечение алгебраического подмногообразия в $V$ с окрестностью образа $K$.

3.10. Неравенства для рода в штейновых поверхностях. Основная идея может быть сформулирована так: вложенные вешественные поверхности в штейновых поверхностях ведут себя так же, как в минимальных кэлеровых поверхностях с $b^{+}>1$. Мы докажем следуюший результат.

Теорема 9. Пусть $S \subset X-$ вложенная ориентированная вещественная поверхность рода g в итейновой поверхности. Предположим, что $S$ не является вложенной сферой с нулевым классом гомологий. Тогда

$$
S^{2}+\left|c_{1}(X) \cdot S\right| \leqslant 2 g-2 .
$$

ДокАЗАТЕЛЬСтво. Мы можем всегда считать, что класс гомологий $S$ отличен от нуля, поскольку доказьваемое неравенство тривиально, если $S$ не сфера и $[S]=0$. В этом случае найдется неособая (некомпактная) комплексная кривая $L \subset X$ с ненулевьм индексом пересечения $L \cdot S \neq 0$. Действительно, на штейновой поверхности группа $H_{2}(X, \mathbb{Z})$ не имеет кручения, и по теореме двойственности Пуанкаре сушествует такой класс $c \in H^{2}(X, \mathbb{Z})$, что $c \cdot S \neq 0$. Поскольку поверхность $X$ штейнова, $c=c_{1}(\mathscr{L})$ для голоморфного линейного расслоения $\mathscr{L}$. Теперь в качестве $L$ можно взять дивизор общего голоморфного сечения расслоения $\mathscr{L}$. 
Воспользовавшись теоремой 8 об алгебраической аппроксимации, мы получим открытое вложение окрестности поверхности $S$ в аффиинное алгебраическое многообразие $V$, при котором $L$ переходит в алгебраическую кривую $C \subset V$, так что $C \cdot S=$ $L \cdot S \neq 0$. Это гарантирует нетривиальность вещественного гомологического класса $S$ после алгебраизации.

Пусть $\bar{V}$ - проективное замыкание многообразия $V$, и пусть $\pi: Y \rightarrow \bar{V}$ - его десингуляризация. Очевидно, $Y$ является компактной алгебраической поверхностью. Обозначим через $H=\pi^{-1}(\bar{V} \backslash V)$ прообраз гиперплоского сечения $\bar{V}$ на бесконечности. Рассмотрим отдельно два случая.

1. Случай $S^{2} \geqslant 0$. Мы имеем два ненулевых класса $[S]$ и $[H]$ в двумерных когомологиях $H^{2}(Y, \mathbb{R})$, причем $[S]^{2} \geqslant 0,[H]^{2}>0$ и $[H] \cdot[S]=0$. Поскольку форма пересечения невырождена, отсюда сразу вытекает, что $b^{+}(Y)>1$. Таким образом, требуемое неравенство следует из теоремы 6.

2. Случай $S^{2}<0$. Применим к вложенной поверхности $S \subset Y$ теорему 7. Заметим для этого, что любая неприводимая кривая в $Y$ либо имеет положительньй индекс пересечения с $H$, либо стягивается в точку отображением $\pi$. По построению неотрицательная линейная комбинация таких кривых не может быть гомологична $\pm S$. Таким образом, неравенство для рода из теоремы 7 справедливо для поверхности $S$.

ЗАмЕчАниЕ 1 . По теореме 4 доказанное неравенство является точньм: удовлетворяющая ему вложенная вешественная поверхность в произвольной комплексной поверхности изотопна вложенной поверхности с базисом штейновых окрестностей. Помимо этого, теорема 4 дает следуюшую чисто геометрическую формулировку теоремы 9: Вложсенная вещественная поверхность в штейновой поверхности, отличная от гомологичной нулю сферы, изотопна вложенной поверхности с фундаментальной системой штейновых окрестностей.

ЗАмЕчАниЕ 2. Часть теоремы, относящаяся к вложенньм поверхностям с неотрицательным самопересечением, справедлива и для не штейновых строго псевдовыпуклых областей. Это следует аналогичным образом (см. [34] и [35]) из алгебраизации таких областей, построенной Лемпертом [26]. Можно также применить теорему Богомолова и де Оливейры [3], согласно которой минимальную строго псевдовьпуклую область можно продеформировать в штейнову область.

ЗАМЕЧАНИЕ 3: ПОГРУЖЕННЫЕ ПОВЕРХНОСТИ. ПредПоложим, что Поверхность $S$ погружена с $\kappa_{+}$положительными и $\kappa_{-}$отрицательньми простьми двойными точками. В этом случае, вложив окрестность $S$ в алгебраическую поверхность $Y$, заменим каждую положительную двойную точку вложенной ручкой и раздуем каждую отрицательную двойную точку. Эта операция увеличит род поверхности на $\kappa_{+}$, но не изменит $S^{2}$ и $c_{1}(Y) \cdot S$. Таким образом, теорема 9 справедлива с заменой $g$ на $g+\kappa_{+}$.

3.11. Топология штейновых поверхностей. На каждом многообразии Штейна $X$ существует строго плюрисубгармоническая функция Морса. Индекс любой критической точки такой функции не превосходит $n=\operatorname{dim}_{\mathbb{C}} X$, и, следовательно, многообразие $X$ допускает разложение на ручки, в котором нет ручек более чем половинной размерности. Элиашберг [9] доказал, что если $n>2$, то существование тако- 
го разложения на ручки на почти комплексном многообразии является достаточным условием сушествования на нем штейновой структуры.

В комплексной размерности 2 это утверждение справедливо только в топологической категории. А именно, Гомпф [15] показал, что открытое ориентированное топологическое четырехмерное многообразие ориентированно гомеоморфно штейновой поверхности тогда и только тогда, когда оно является внутренностью тела с ручками без ручек индекса $>2$. Более того, таким образом может быть реализована любая почти комплексная структура.

Результат Гомпфа использует ручки Кассона, и потому естественно ожидать, что подлежащие гладкие многообразия получающихся штейновых поверхностей будут в некотором смысле “экзотическими”. Мы хотим сравнить эти результаты с теоремой 9 .

Пусть $H_{n}$ - это ориентированное вещественное двумерное векторное расслоение с числом Эйлера $n \in \mathbb{Z}$ на двумерной сфере. Обозначим через $S_{n}$ нулевое сечение $H_{n}$. По теоремам 9 и 4 гладкое ориентированное многообразие $H_{n}$ допускает структуру штейновой комплексной поверхности $X_{n}$, для которой $c_{1}\left(X_{n}\right) \cdot S_{n}=k$ $(k \equiv n \bmod 2)$, в том и только том случае, если $n+|k| \leqslant-2$. В частности, при $n \geqslant-1$ многообразие $H_{n}$ не может быть ориентированно диффеоморфно штейновой поверхности, а при $-1 \leqslant n \leqslant 1$ оно не диффеоморфно штейновой поверхности ни при каком выборе ориентации.

С другой стороны, теорема 3.3 из [15] утверждает, что многообразие $H_{n}$ ориентированно гомеоморфно штейновой поверхности $V_{n}$, не содержащей гомологически нетривиальных гладко вложенных сфер и реализуюшей любую заданную почти комплексную структуру.

В частности, многообразие $H_{-1}$ одновременно

1) ориентированно диффеоморфно строго псевдовьпуклой области (а именно, окрестности исключительной кривой раздутия),

2) ориентированно гомеоморфно штейновой поверхности,

3) не диффеоморфно штейновой поверхности.

В работе [15] имеется немало других красивых примеров. Так, существуют “фальшивые" штейновы $\mathbb{R}^{4}$, гомеоморфные, но не диффеоморфные стандартному $\mathbb{R}^{4}$. Как отмечено в [15], эти многообразия диффеоморфны областям в $\mathbb{R}^{4}$. Интересно было бы понять, а биголоморфны ли они областям в $\mathbb{C}^{2}$ ?

\section{$\S$ 4. Оболочки голоморфности вещественньх поверхностей в комплексных поверхностях}

В этом параграфе мы применим неравенства для рода вложенных вешественных поверхностей в штейновых поверхностях к изучению оболочек голоморфности областей в различных комплексных поверхностях. В том числе, в п. 4.1 доказывается сформулированная во введении гипотеза Витушкина о голоморфных функциях в окрестности двумерной сферы в $\mathbb{C P}^{2}$.

Основная идея весьма проста. Предположим, что мы умеем решать проблему Леви на заданной комплексной поверхности $X$, т.е. можем указать условия, при которых 
оболочка голоморфности области $U \subset X$ штейнова. Тогда применение теоремы 9 приводит к топологическим ограничениям на вешественные поверхности в $U$. Результаты пाп. 4.1-4.3 взяты из работы [34].

4.1. Вещественные поверхности в $\mathbb{C P}^{2}$ и гипотеза Витушкина о сфере. Пусть $S \subset \mathbb{C P}^{2}$ - вложенная ориентированная вешественная поверхность в комплексной проективной плоскости. Определим степень $d=S \cdot\left[\mathbb{C P}^{1}\right]$ поверхности $S$ как ее индекс пересечения с проективными прямыми. Мы можем так выбрать ориентацию на $S$, что $d \geqslant 0$. Заметим, что поверхность $S$ гомологична нулю тогда и только тогда, когда $d=0$.

Для вложенной поверхности степени $d$ мы имеем $S^{2}=d^{2}$ и $c_{1}\left(\mathbb{C P}^{2}\right) \cdot S=3 d$. В частности, если $S$ - это неособая комплексная кривая, то формула для рода имеет классический вид $g(S)=g_{\mathbb{C}}(d)=\frac{1}{2}\left(d^{2}-3 d+2\right)$.

ТеОрема 10. Пусть $S \subset \mathbb{C P}^{2}$ - вложсенная ориентированная вещественная поверхность положстельной степени $d>0$ и рода g. Если в окрестности $S$ существует непостоянная голоморфная функция, то выполнено неравенство

$$
g \geqslant \frac{1}{2}\left(d^{2}+3 d+2\right)=g_{\mathbb{C}}(d)+3 d .
$$

Обратно, если поверхность $S$ удовлетворяет этому неравенству, то она изотопна вложенной поверхности с базисом штейновых окрестностей.

СЛЕДСТВИЕ: ГИПОТЕЗА ВИТУШКИНА. Любая голоморфная функция в окрестности негомологичной нулю вложенной двумерной сферы в $\mathbb{C P}^{2}$ постоянна.

ДокаЗАтЕЛЬСтво теоРемы. Рассмотрим окрестность $U \supset S$. Если в $U$ существует непостоянная голоморфная функция, то по теореме 1 оболочка голоморфности $\widetilde{U} \supset U$ является многообразием Штейна, поскольку она не может совпадать с $\mathbb{C P}^{2}$. Следовательно, требуемое неравенство немедленно следует из теоремы 9.

Если же это неравенство выполнено, то по теореме 4 действительно сушествует изотопная $S$ вложенная поверхность с базисом штейновых окрестностей.

4.2. Поверхности в $\mathbb{C P}^{1} \times \mathbb{C P}^{1}$. Рассмотрим ориентированную вешественную поверхность $S \subset \mathbb{C P}^{1} \times \mathbb{C P}^{1}$ и определим ее “бистепень" $d=\left(d_{1}, d_{2}\right)$ как пару индексов пересечения с вертикальными и горизонтальными прямьми. Тогда $c_{1}\left(\mathbb{C P}^{1} \times \mathbb{C P}^{1}\right) \cdot S=$ $2\left(d_{1}+d_{2}\right)$ и $S^{2}=2 d_{1} d_{2}$.

Неособая комплексная кривая $C \subset \mathbb{C P}^{1} \times \mathbb{C P}^{1}$ имеет "положительную" бистепень $d \in \mathbb{Z}_{+}^{2} \backslash\{0\}$. Род кривой $C$ дается формулой $g_{\mathbb{C}}(d)=d_{1} d_{2}-d_{1}-d_{2}+1$.

ТеОрема 11. Пусть $S \subset \mathbb{C P}^{1} \times \mathbb{C P}^{1}$ - вложсенная ориентированная вещественная поверхность ненулевой бистепени $d=\left(d_{1}, d_{2}\right) \in \mathbb{Z}^{2} \backslash\{0\}$ и рода g. Если в окрестности поверхности $S$ существует непостоянная голоморфная функиия, то либо

$$
g \geqslant d_{1} d_{2}+\left|d_{1}+d_{2}\right|+1, \quad d_{1} d_{2} \neq 0
$$


либо $d_{1} d_{2}=0$ и оболочка голоморфности $S$ содерәсит вертикальную или горизонтальную прямую.

Если поверхность $S$ удовлетворяет неравенству (**), то она изотопна вложенной поверхности с базисом итейновых окрестностей.

В частности, невозможно продеформировать неособую комплексную кривую в $\mathbb{C P}^{1} \times \mathbb{C P}^{1}$, отличную от вертикальной или горизонтальной прямой, во вложенную вещественную поверхность с непостоянными голоморфными функциями в окрестности.

ДоКАЗАТЕЛЬСТВо. Согласно теореме 1 оболочка голоморфности любой области $U \subset \mathbb{C P}^{1} \times \mathbb{C P}^{1}$ либо штейнова, либо содержит один из сомножителей. Заметим, кроме того, что если оболочка $\widetilde{U}$ содержит, скажем, вертикальную прямую и пересекает все остальные вертикальные прямые, то она совпадает с $\mathbb{C P}^{1} \times \mathbb{C P}^{1}$ по лемме Хартогса из п. 1.6 .

Остается применить теоремы 9 и 4 точно так же, как и в доказательстве предыдущей теоремы.

\section{3. Произведения сферы Римана на открытую риманову поверхность.} Рассмотрим прямое произведение $X=\mathbb{C P}^{1} \times Y$, где $Y$ - некомпактная связная риманова поверхность. Напомним, что $H^{2}(Y, \mathbb{Z})=0$ и по формуле Кюннета $H^{2}(X, \mathbb{Z}) \cong$ $H^{2}\left(\mathbb{C P}^{1}, \mathbb{Z}\right) \cong \mathbb{Z}$. Назовем степенью вложенной вещественной поверхности $S \subset X$ топологическую степень $d=\operatorname{deg} \pi_{\mathbb{C P}^{1}}$ ее проекции на сомножитель $\mathbb{C P}^{1}$. Тогда $S^{2}=0$ и $c_{1}(X) \cdot S=2 d$. Заметим также, что степень $d \geqslant 0$ при соответствующем выборе ориентации на $S$.

По классической теореме Бенке-Штейна одномерное комплексное многообразие $Y$ штейново. Таким образом, теорема 1 дает следуюшее описание оболочек голоморфности над $X$. Оболочка области $U \subset X$ либо штейнова, либо содержит некоторый слой $\mathbb{C P}^{1} \times\{y\}$. Во втором случае все голоморфные функции на $U$ постоянны вдоль $\mathbb{C P}^{1}$, и, следовательно, оболочка голоморфности имеет вид $\mathbb{C P}^{1} \times \pi_{Y}(U)$.

Теперь, используя то же самое рассуждение, что и вьше, мы получаем следующий результат.

ТЕОРема 12. Пусть $S \subset \mathbb{C P}^{1} \times Y$ - вложенная ориентированная вещественная поверхность положительной степени $d>0$ и рода $g$. Eсли $g<d+1$, то любая голоморфная функиия в окрестности $S$ продолжается в окрестность мнозества $\mathbb{C P}^{1} \times \pi_{Y}(S)$.

Если $g \geqslant d+1$, то поверхность $S$ изотопна вложсенной поверхности с базисом штейновых окрестностей.

4.4. Лемма Чирки-Хартогса. Пусть $F: \mathbb{C P}^{1} \rightarrow Y$ - гладкое отображение из сферы Римана в некомпактную риманову поверхность. График этого отображения $\Gamma_{F}$ представляет собой вложенную сферу в $X=\mathbb{C P}^{1} \times Y$ степени 1 (проекция $\Gamma_{F}$ на $\mathbb{C P}^{1}$ взаимно однозначна). Таким образом, по теореме 12 любая голоморфная функиия в окрестности $\Gamma_{F}$ продоләсается в окрестность множества $\mathbb{C P}^{1} \times F\left(\mathbb{C P}^{1}\right)$. 
Этот результат был впервые получен Чиркой [5] с помощью модификации основанного на теории псевдоголоморфных кривых метода, предложенного Ивашковичем и Шевчишиньп [17]. Чирка использовал эту теорему об аналитическом продолжении для доказательства обобщения леммы Хартогса из п. 1.6, высказанного в качестве гипотезы Андреем Домриньм.

Пусть $f: \bar{\Delta} \rightarrow \bar{\Delta}$ - непрерывная функция в единичном круге, для которой $\|f\| \leqslant 1$. Можно рассмотреть следуюшее обобщение фигуры Хартогса:

$$
D_{f}=\Gamma_{f} \cup\left\{(z, w) \in \mathbb{C}^{2}|| z|=1,| w \mid \leqslant 1\right\} \subset \mathbb{C}^{2} .
$$

Классическая фигура Хартогса получается при $f \equiv 0$.

ТЕорема 13 (Е.М. Чирка). Любая голоморфная функиия в окрестности $D_{f}$ голоморфно продолжсается в окрестность единичного бидиска $D$.

ДокАЗАТЕльство. Заметим сразу, что можно считать функцию $f$ гладкой и удовлетворяющей условию $\left.f\right|_{\partial \Delta} \equiv 0$. Такая функция $f$ тривиально продолжается до гладкого отображения $F: \mathbb{C P}^{1} \rightarrow \mathbb{C}$.

Пусть теперь $h$ - голоморфная функция на $D_{f}$. Раскладывая $h$ в ряд Лорана по переменной $z$, мы получаем разложение $h=h_{+}+h_{-}$, где функция $h_{+}$голоморфна в бидиске $D$, а функция $h_{-}$- на "бесконечном" бидиске $D_{\infty}=\left(\mathbb{C P}^{1} \times \Delta\right) \backslash D$. Положим $\psi=h-h_{+}$на $D_{f}$ и $\psi=h_{-}$на $D_{\infty}$. Тогда функция $\psi$ голоморфна в окрестности графика отображения $F$, содержащей множество $\{|z|=1,|w| \leqslant 1\}$. По теореме 12 функция $\psi$ голоморфно продолжается на $\mathbb{C P}^{1} \times \Delta$. В частности, $\psi$ продолжается на $D$, и тем же свойством обладает функция $h=\psi+h_{+}$.

4.5. Оболочки симплектических поверхностей и теорема Ивашковича-Шевчишина. Пусть $X$ - кэлерова комплексная поверхность с кэлеровой формой $\omega$. Вложенная вешественная поверхность $S \subset X$ называется симплектической, если положительно ограничение $\left.\omega\right|_{S}>0$. Такая поверхность $S$ псевдоголоморфна относительно подходящей почти комплексной структуры $J$, согласованной с $\omega$. В частности, поверхность $S$ удовлетворяет формуле для рода $2 g-2=S^{2}-c_{1}(X) \cdot S$. Заметим еще, что вешественньй гомологический класс симплектической поверхности заведомо отличен от нуля.

Точно так же, как для комплексных кривых в п. 3.6, мы видим, что симплектическая поверхность не удовлетворяет неравенству $2 g-2 \geqslant S^{2}+\left|c_{1}(X) \cdot S\right|$ из теоремы 9 тогда и только тогда, когда $c_{1}(X) \cdot S>0$. Мы заключаем, что оболочка голоморфности симплектической поверхности $S \subset X$, для которой $c_{1}(X) \cdot S>0$, не может бъть штейновой. Например, любая голоморфная функция в окрестности симплектической поверхности в $\mathbb{C P}^{2}$ постоянна. Отметим, что эти результаты справедливы и для погруженных симплектических поверхностей с положительными двойными точками по замечанию 3 после теоремы 9.

Нетривиальность оболочек симплектических сфер в “положительных" кэлеровых поверхностях была обнаружена Ивашковичем и Шевчишиньм [17]. В частности, они доказали гипотезу Витушкина для симплектических сфер в $\mathbb{C P}^{2}$. Метод, предложенный и развитый в работах [17], [18] и [19], использует теорию псевдоголоморфных кривых Громова и обобшение принципа непрерывности из п. 1.6. 
Сформулируем основной результат работы [18]. Заметим сначала, что для области $U \subset X$ в комплексном многообразии можно наряду с оболочкой голоморфности определить также оболочку мероморфности или, более общим образом, оболочку мероморфности относительно некоторого многообразия $Y$. Это максимальная область $(\widetilde{U}, \pi)$ над многообразием $X$, на которую мероморфно продолжается любое мероморфное отображение $f: U \rightarrow Y$.

Нам понадобится также следующее свойство вьпуклости, приспособленное для применений принципа непрерывности. Комплексное эрмитово многообразие $(X, h)$ назьвается диск-выпуклыцм, если для любой последовательности $\left(C_{n}, u_{n}\right)$ стабильных комплексных кривых в $X$, параметризованных одной и той же вешественной поверхностью $\Sigma$ и таких, что

1) $C_{n}$ неприводимы при всех $n$, и границы $\partial C_{n}$ непусты;

2) площади $\operatorname{area}_{h}\left[u_{n}\left(C_{n}\right)\right]$ равномерно ограничены;

3) $\left(C_{n}, u_{n}\right)$ сходятся в окрестности границ $\partial C_{n}$,

существует компакт $K \Subset X$, содержаший все $u_{n}\left(C_{n}\right)$.

Легко видеть, что компактные многообразия и многообразия Штейна диск-выпуклы. Более общим образом, доказанньй в [18] принцип непрерьвности гласит, что для любой области в диск-выпуклой комплексной поверхности ее оболочка мероморфности со значениями в диск-вьпуклом многообразии также является диск-выпуклой.

Tеорема 14. Пусть $S \subset X-$ погруженная симплектическая сфера с положительными двойныцми точками в диск-выпуклой кәлеровой поверхности. Предположим, что $c_{1}(X) \cdot S>0$. Тогда оболочка голоморфности любой окрестности $S$ относительно диск-выпуклого многообразия $Y$ содержит компактную комплексную рациональную кривую.

Замечание 1. Теорема Чирки 13 следует из этого результата. Действительно, графии гладкого отображения $F: \mathbb{C P}^{1} \rightarrow \mathbb{C}$ является симплектической сферой относительно подходяшим образом выбранной кэлеровой формы на $\mathbb{C P}^{1} \times \mathbb{C}$.

ЗАмЕчАниЕ 2. Для симплектических сфер эта теорема сильнее приведенных выше следствий теоремы 9 даже в случае оболочек голоморфности. Вообше говоря, не штейнова оболочка может не содержать компактных комплексных кривых.

\section{$\S 5$. Двумерные сферы и диски в $\mathbb{C}^{2}$}

В этом параграфе мы в основном применяем теорему 9 к вложенным сферам в пространстве $\mathbb{C}^{2}$ и его раздутиях. Это позволяет доказать, что вложенная двумерная сфера в $\mathbb{C}^{2}$ гомологична нулю в своей оболочке голоморфности. Мы также доказываем еше одну известную гипотезу Витушкина о невозможности приклеить аналитический диск снаружи к диффеоморфной шару строго псевдовыпуклой области в $\mathbb{C}^{2}$ и получаем ее обобшения и аналоги.

5.1. Оболочки двумерных сфер. По теореме 9 вложенная сфера $S$ с индексом самопересечения $S^{2}=0$ в штейновой комплексной поверхности $X$ обязательно гомологична нулю в $H_{2}(X, \mathbb{Z})$. Для вложенной сферы $S \subset \mathbb{C}^{2}$ условие $S^{2}=0$ выполнено, 
поскольку вообще $H_{2}\left(\mathbb{C}^{2}, \mathbb{Z}\right)=0$. Таким образом, сфера $S$ гомологична нулю в любой штейновой области над $\mathbb{C}^{2}$, содержащей ее окрестность. Поскольку оболочка голоморфности любой области в $\mathbb{C}^{2}$ штейнова, мы получаем следуюший результат [35].

Теорема 15. Вложсенная двумерная сфера $S \subset \mathbb{C}^{2}$ гомологична нулю в оболочке голоморфности любой своей окрестности $U \supset S$.

Рассмотрим, например, голоморфную 2-форму $\omega=f(z, w) d z \wedge d w, f \in \mathscr{O}(U)$, определенную в окрестности $U \supset S$. Очевидно, $\omega$ голоморфно продолжается на оболочку $\widetilde{U} \supset U$. Но сфера $S$ гомологична нулю в $\widetilde{U}$, и поскольку голоморфные формы замкнуты, мы видим, что $\int_{S} \omega=0$.

Этот результат позволяет получить некоторые ограничения на приближения голоморфными функциями на вложенных сферах. Для компакта $K \subset \mathbb{C}^{2}$ обозначим через $C(K)$ пространство всех непрерывных комплекснозначных функций на $K$, а через $A(K)$ - подпространство функций, допускающих равномерную на $K$ аппроксимацию голоморфными функциями в окрестностях $K$.

СледСТВИЕ. Пусть $S \subset \mathbb{C}^{2}$ - вложсенная двумерная сфера, тогда каждая функиия $f \in A(S)$ удовлетворяет “условиям моментов"

$$
\int_{S} f h d z \wedge d w=0, \quad h \in A(S) .
$$

В частности, $A(S)$ имеет бесконечную коразмерность в $C(S)$.

Напомним, что согласно п. 2.5 существуют вложенные вещественные поверхности положительного рода в $\mathbb{C}^{2}$, имеющие только гиперболические комплексные точки и допускаюшие базис штейновых окрестностей. Можно показать, что для таких поверхностей $A(S)=C(S)$.

На самом деле, хороший элементарньй пример такого поведения доставляет стандартный тор $T=\{|z|=1,|w|=1\} \subset \mathbb{C}^{2}$. Это вполне вешественное подмногообразие, не гомологичное нулю в своей трубчатой штейновой окрестности. Форма $\omega=\frac{d z}{z} \wedge \frac{d w}{w}$ голоморфна в окрестности $T$, но $\int_{T} \omega=-4 \pi^{2}$. Любую непрерьвную функцию на $T$ можно равномерно приблизить полиномами Лорана от $(z, w)$ (можно взять полиномы, соответствуюшие суммам Фейера ряда Фурье этой функции).

Отметим также, что доказанная теорема, вообе говоря, неверна для погруженныx сфер. Действительно, приспосабливая результаты $\S 2$ для погруженных поверхностей (нужно заменить $S^{2}$ в формулах Лая на число Эйлера нормального расслоения), нетрудно видеть, что сушествует погруженная вполне вещественная двумерная сфиера в $\mathbb{C}^{2}$ с единственной положительной двойной точкой. Эта сффера имеет базис штейновых окрестностей, в которых она не гомологична нулю. В то же время, для погруженных сфер с отрицательными двойньми точками наша теорема справедлива в силу замечания 3 к теореме 9 .

5.2. Заполнение аналитическими дисками. Предположение, что оболочка голоморфности вложенной сферы в $\mathbb{C}^{2}$ должна быть нетривиальна, исходит из рассмотрения стандартной двумерной сферы $S=\left\{|z|^{2}+|w|^{2}=1, \operatorname{Im} w=0\right\}$. Эта 
сфера, очевидно, ограничивает семейство вложенных аналитических дисков $D_{t}=$ $\left\{|z|^{2}+|w|^{2}=1, \operatorname{Re} w=t\right\}, t \in[-1,1]$. По принципу непрерьвности любая голоморфная функция в окрестности сферы $S$ голоморфно продолжается вдоль семейства $D_{t}$ в окрестность Леви-плоского трехмерного шара, натянутого на $S$.

Стандартная сфера имеет две эллиптические комплексные точки противоположного знака и лежит в границе стандартного четырехмерного шара в $\mathbb{C}^{2}$. Бишоп [2] обнаружил, что в окрестности любой эллиптической комплексной точки на вешественной поверхности существует начинающееся из этой точки непрерьвное семейство вложенных непересекающихся аналитических дисков с границами на данной поверхности. Позже было показано, что если поверхность лежит в границе строго псевдовыпуклой области, то глобальное поведение таких семейств можно контролировать даже при наличии гиперболических точек (см. обзор [1]). В частности, этим методом Элиашберг [8] доказал неравенства для рода вешественных поверхностей в строго псевдовыпуклых границах. Приведем для сравнения с теоремой 15 наиболее общий результат о заполнении сфер в $\mathbb{C}^{2}$ аналитическими дисками, полученньй Кружилинытм [24].

Теорема 16. Пусть $U$ - строго псевдовьпуклая область в $\mathbb{C}^{2}$, и пусть $S$ вложенная в $\partial U$ двумерная сфера класса $C^{6}$, вполне вещественная всюду, кроме конечного множсества точек, состоящего из $K$ гиперболических и $K+2$ эллиптических комплексных точек. Тогда существуют:

1) гладкая область $W \subset \mathbb{R}^{3}\left(x_{1}, x_{2}, x_{3}\right)$ с границей, диффеоморфной $S$, и такая, что (а) все множества уровня функиии $x_{3}$ на $W$ являются конечными объединениями топологических дисков, и (б) $x_{3}$ является функиией Морса на $\partial W$ с $K+2$ экстремумами и $K$ седлами;

2) непрерывное ингективное отображсение $\Psi: W \rightarrow U$, переводящее $\partial W$ в сферу $S$, әкстремумы и седла функиии $x_{3}$ на $\partial W$ в әллиптические $и$ гиперболические точки $S$ и связные компоненты множеств $\{x \in W$ : $x_{3}=$ const $\}$ в гладкие аналитические диски.

Мнохество $\Psi(W)$ является оболочкой голоморфности сферы $S$, а также ее въпуклой оболочкой относительно алгебры $A(\bar{U})$.

ЗАмечАниЕ 1. Тот факт, что число эллиптических точек на вложенной сфере на 2 больше числа гиперболических, следует из формул Лая. Отметим, однако, что невозможно использовать теорему 3 о сокрашении внутри вешественной гиперповерхности $\partial U$ для устранения излишних пар комплексных точек.

ЗАмЕчАниЕ 2. Условие, что вложенная сфера содержится в границе строго псевдовьпуклой области, не может быть опушено. Так, Форнесс и Ма [11] явно указали незаузленную двумерную сферу в $\mathbb{C}^{2}$, имеющую две эллиптические комплексные точки, для которой семейства дисков Бишопа, начинаюшиеся из этих точек, не образуют трехмерной поверхности.

5.3. Приклеивание аналитических дисков к строго псевдовыпуклым областям. Пусть $U \subset X$ - относительно компактная область с гладкой границей на комплексном многообразии. Будем говорить, что вложенный аналитический диск 
$D \subset X$, гладкий вплоть до гранишы, приклеен (снаружи) к области $U$, если $D \subset X \backslash U$ и $\partial D \subset \partial U$. Мы всегда будем считать, что диск $D$ трансверсален $\partial U$.

Нас интересует ситуация, когда область $U$ строго псевдовьпукла. Например, диск $\{|z| \leqslant 1, w=0\} \subset \mathbb{C}^{2}$ приклеен снаружи к $\varepsilon$-окрестности окружности $\{|z|=1+\varepsilon$, $w=0\}$. Заметим, что граница этого диска не гомологична нулю в $U$.

Более поучительньй пример можно построить следующим образом. Рассмотрим вполне вешественньй тор $T \subset \mathbb{C}^{2}$. Обрашая доказательство теоремы 3 , создадим эллиптическую и гиперболическую точку на торе $T$. Заменим окрестность эллиптической точки аналитическим диском и заметим, что этот диск приклеен к строго псевдовыпуклой окрестности остальной части тора. Гранища этого диска ограничивает вещественную поверхность с одной ручкой внутри области. Важное наблюдение (принадлежашее Форстнеричу [12]) состоит в том, что по формулам Лая такую конструкцию нельзя осуществить со сферой. Точнее говоря, невозможно так склеить вдоль общей гранишы аналитический диск и диск без эллиптических точек, чтобы они не имели общих внутренних точек.

Мы обобшим эти результаты, следуя [35], и покажем, что граница аналитического диска, приклеенного к строго псевдовыпуклой области в $\mathbb{C}^{2}$, не может быть срезана гладким диском внутри этой области.

ТЕОРема 17. Пусть аналитический диск $D$ приклеен $к$ строго псевдовыпуклой области $U$ в штейновой поверхности $X$ с $H_{2}(X, \mathbb{R})=0$. Тогда не существует гладкого диска в области $U$ с той жсе границей.

ДокАЗАТЕЛЬСтво. Предположим, что сушествует гладкий диск в $U$ с границей $\partial D$. Сглаживая объединение этих двух дисков, мы получим вложенную сферу $S \subset X$. Представим себе, что нам удалось так подклеить трубчатую окрестность диска $D$ к области $U$, что получившаяся область штейнова. Тогда сфера $S$ не гомологична нулю в этой области, и получается противоречие с теоремой 9 . Следующий основной пример сначала показьвает, что такая подклейка окрестности невозможна, но затем дает решение этой проблемы.

Рассмотрим стандартный диск $D=\{w=0,|z| \leqslant 1\}$ в $\mathbb{C}^{2}$ и строго псевдовыпуклый "воротник" $C=\left\{|w|^{2}+(|z|-a)^{2} \leqslant a-1,|z| \leqslant 1+\varepsilon\right\}$ для некоторого $a>1$ и $\varepsilon>0$. Если приклеить малую окрестность диска $D$ к воротнику, то внутренность полученного множества содержит фигуру Хартогса с подходящими “стенками” и не может быть штейновой областью. Однако мы можем построить штейнову окрестность, "вырожденную" в начале координат.

Заметим для этого, что в логарифмических координатах $\xi=\ln |z|, \eta=\ln |w|$ область с внешней стороны воротника $C$ соответствует подграфику гладкой выпуклой вверх функции $\eta=f(\xi), \xi \in(0, \ln (1+\varepsilon)]$. Ось $\eta$ является, очевидно, вертикальной асимптотой для $f$. Выберем точку $\xi_{0} \in(0, \ln (1+\varepsilon)]$ так, что наклон касательной $T_{\xi_{0}} \Gamma_{f}$ является натуральным числом $n \geqslant 1$, и заменим график функции $f$ этой касательной при всех $\xi \leqslant \xi_{0}$. Подграфик этой новой функции определяет логарифмически вьпуклую и, значит, штейнову окрестность множества $D \backslash\{0\}$. В окрестности начала координат эта область имеет вид $|w| \leqslant K|z|^{n}, K>0$.

Заметим теперь, что если раздуть начало координат в $\mathbb{C}^{2}$, т.е. выполнить замену координат $z=z^{\prime}, w=w^{\prime} z^{\prime}$, то собственный прообраз построенной вырожденной 
окрестности примет вид $\left|w^{\prime}\right| \leqslant K\left|z^{\prime}\right|^{n-1}$. Таким образом, после $n$ раздутий мы получим настояшую окрестность собственного прообраза $D \cup C$. По построению эта окрестность локально псевдовьпукла и пересекает только последнюю исключительную кривую раздутий по диску. Следовательно, она штейнова по замечанию после теоремы Такеучи 2.

Ситуация теоремы сводится к разобранной модели подходящей заменой координат в вырожденной окрестности диска $D$ и шевелением области $U$. Итак, мы получаем следуюшую конструкцию штейновых окрестностей.

ЛЕмма 2. Преположим, что аналитический диск $D$ приклеен снаружи $к$ строго псевдовыпуклой области $U$ в итейновой поверхности $X$. Тогда собственный прообраз $D \cup U$ после нескольких раздутий в точке $p \in D$ содержится в штейновой области.

Чтобы завершить доказательство теоремы, вспомним, что $H_{2}(X, \mathbb{R})=0$, а потому $S^{2}=0$ и $c_{1}(X) \cdot S=0$. Значит, для собственного прообраза $\widetilde{S} \subset \widetilde{X}$ сферы $S$ после $n \geqslant 1$ раздутий в точке $p \in S$ мы имеем $\widetilde{S}^{2}=-n$ и $\left|c_{1}(\widetilde{X}) \cdot \widetilde{S}\right|=n$, что противоречит неравенству из теоремы 9.

СЛЕДСТВИЕ: ГИПОТЕЗА ВИТУШКИНА. Нельзя приклеить аналитический диск снаружи к диффеоморфной шару строго псевдовыпуклой области в $\mathbb{C}^{2}$.

ДокАЗАТЕльство. Идея в том, чтобы “отразить” диск внутрь области с помощью инверсии.

Пусть $U \subset \mathbb{C}^{2}$ - диффеоморфная шару строго псевдовьпуклая область в $\mathbb{C}^{2}$. Точнее говоря, мы предполагаем, что существует диффеоморфизм многообразий с краем $\psi: \bar{U} \rightarrow \bar{B}$. Тогда $\psi$ продолжается до диффеоморфизма $\Psi$ пространства $\mathbb{C}^{2}$, переводяшего $U$ в стандартный шар $B$.

Если к области $U$ снаружи приклеен аналитический диск, то его образ $\Psi(D)$ представляет собой гладкий диск, приклеенный к шару $B$. Применяя инверсию относительно $\partial B$, мы получаем гладкий диск $D^{\prime}$ внутри $B$ с той же границей. Теперь прообраз $\Psi^{-1}\left(D^{\prime}\right)$ - это гладкий диск внутри области $U$ с границей $\partial D$, что противоречит предыдущей теореме.

5.4. Приклеивание вполне вещественных дисков. Результаты предыдушего пункта имеют аналоги для вполне вешественных дисков, приклеенных к строго псевдовыпуклым областям по лежсандровым кривым.

Напомним, что кривая $\gamma \subset \partial U$ в строго псевдовыпуклой границе назьвается лежандровой, если она касательна к распределению комплексных касательных $T^{c} \partial U=$ $T \partial U \cap i T \partial U$. Элиашберг [9] показал, что если вполне вешественньй диск $D$ приклеен к области $U$ по лежандровой кривой, то трубчатую окрестность диска $D$ можно так подклеить к области $U$, что получится строго псевдовьпуклая область (см. также [37]). Напомним также, что по замечанию 2 после теоремы 9 ограничения на вещественные поверхности с неотрицательньм индексом самопересечения имеют тот же вид для необязательно штейновых строго псевдовьпуклых областей. Таким образом, мы получаем следующие результаты. 
ТЕОРема 18. Пусть вполне вещественный диск $D$ приклеен по лежсандровой кривой к строго псевдовыпуклой области $U$ в комплексной поверхности $X$ с неотрицательной формой пересечения на $H_{c}^{2}(X, \mathbb{R})=0$. Тогда $\partial D$ не является границей гладкого диска в $U$.

СлЕДСТВИЕ. Невозможно приклеить вполне вещественный диск по лежандровой кривой $к$ диффеоморфной шару строго псевдовыпуклой области в $\mathbb{C}^{2}$.

Связь между теоремами 17 и 18 можно пояснить с помощью следуюшего геометрического результата Смирнова и Чирки [39].

Рассмотрим единичньй шар $B \subset \mathbb{C}^{2}$ и проходяшую через начало координат двумерную вешественную плоскость $L$. Множество $B \cup L$ полиномиально вьпукло (и допускает базис штейновых окрестностей) тогда и только тогда, когда плоскость $L$ является либо комплексной прямой, либо лагранжевой плоскостью. Второе условие эквивалентно тому, что кривая $L \cap \partial B$ лежандрова.

Пополним $\mathbb{C}^{2}$ до $\mathbb{C P}^{1} \times \mathbb{C P}^{1}$. Замыкание комплексной прямой $L=\{w=0\}$ определяет приклеенный к шару $B$ аналитический диск $\bar{L} \backslash B$, граница которого срезана диском $B \cap L$. Любая раздутая окрестность $\bar{L} \cup B$ содержит собственный прообраз комплексной кривой $\bar{L}$ и потому не является штейновой областью. Лемма 2 не применима, поскольку сама поверхность $\mathbb{C P}^{1} \times \mathbb{C P}^{1}$ не штейнова.

Аналогичным образом, замыкание лагранжевой плоскости $L=\{w=\bar{z}\}$ определяет вполне вешественный диск, приклеенный к шару $B$ по лежандровой кривой, ограничиваюшей диск в $B$. Однако двумерная сфера $S=\bar{L}$, образованная этими двумя дисками, вполне вешественна, и по формулам Лая $S^{2}=-2$ и $c_{1} \cdot S=0$, что не противоречит теореме 9. Теорема 18 здесь не применима, поскольку форма пересечения на $H^{2}\left(\mathbb{C P}^{1} \times \mathbb{C P}^{1}, \mathbb{R}\right)$ имеет сигнатуру $(+,-)$.

Если же пополнить $\mathbb{C}^{2}$ до $\mathbb{C P}^{2}$, где применима теорема 18 , то замькание лагранжевой плоскости даст вешественную проективную плоскость $\mathbb{R P}^{2} \subset \mathbb{C P}^{2}$, и к шару $B$ по лежандровой окружности будет приклеен уже не диск, а лист Мёбиуса.

\section{СПИСОК ЛИТЕРАТУРЫ}

[1] Беннекен Д. Симплектическая топология, голоморфная выпуклость и контактные структуры (по Я. Элиашбергу, Д. Макдаффф и др.) // Труды семинара Бурбаки за 1990 г. М.: Мир, 1996. С. 212-242.

[2] Bishop E. Differentiable manifolds in complex Euclidean space // Duke Math. J. 1965. V. 32. P. $1-22$.

[3] Bogomolov F. A., de Oliveira B. Stein small deformations of strictly pseudoconvex surfaces // Birational Algebraic Geometry. Conf. in memory of W.-L. Chow. Contemp. Math. V. 207: AMS, 1996. P. 25-41.

[4] Bohr Ch. Embedded surfaces and almost complex structures // Proc. Amer. Math. Soc. (to appear).

[5] Чирка Е. М. Обобщенная лемма Гартогса и нелинейное $\bar{\partial}$-уравнение // Комплексный анализ в современной математике. Сб. посв. памяти Б. В. Шабата. М.: Фазис, 1998. С. 19-30.

[6] Chirka E. M., Stout E. L. A Kontinuitätssatz. Banach Center Publ. V. 31. Warsaw, 1995.

[7] Demailly J.-P., Lempert L., Shiffman B. Algebraic approximations of holomorphic maps from Stein domains to projective manifolds // Duke Math. J. 1994. V. 76. № 2. P. 333-363. 
[8] Eliashberg Ya. Filling by holomorphic discs and its applications // Geometry of Low-Dimensional Manifolds. (London Math. Soc. Lect. Notes. V. 151). London, 1990. P. 45-72.

[9] Eliashberg Ya. Topological characterization of Stein manifolds of dimension $>2 / /$ Internat. J. Math. 1990. V. 1. P. 29-46.

[10] Fintushel R., Stern R. Immersed spheres in 4-manifolds and the immersed Thom conjecture // Turkish J. Math. 1995. V. 19. № 2. P. 145-157.

[11] Fornaess J.E., Ma D. A 2-sphere in $\mathbb{C}^{2}$ that cannot be filled in with analytic disks // Internat. Math. Res. Notices 1995. № 1. P. 17-22.

[12] Forstnerič F. Complex tangents of real surfaces in complex surfaces // Duke Math. J. 1992. V. 67. № 2. P. 353-376.

[13] Fujita R. Domaines sans point critique intérieur sur l'espace projectif complexe // J. Math. Soc. Japan. 1963. V. 15. № 4. P. 443-473.

[14] Fujita R. Domaines sans point critique intérieur sur l'espace produit // J. Math. Kyoto Univ. 1965. V. 4. №3. P. 493-514.

[15] Gompf R. E. Handlebody construction of Stein surfaces // Ann. of Math. 1998. V. 148. P. 619-693.

[16] Грауэрт Э. О проблеме Леви и вложении вещественно-аналитических многообразий // Математика. 1960. Т. 4. №3. С. 29-40.

[17] Ivashkovich S. M., Shevchishin V. V. Pseudo-holomorphic curves and envelopes of meromorphy of two-spheres in $\mathbb{C P}^{2} / /$ Preprint. Bochum, 1995.

[18] Ивашкович С. М., Шевчишин В. В. Деформации некомпактных комплексных кривых и оболочки мероморфности сфер // Матем. сб. 1998. Т. 189. №9. С. 23-60.

[19] Ivashkovich S. M., Shevchishin V. V. Structure of the moduli space in the neighbourhood of a cusp curve and meromorphic hulls // Invent. Math. 1999. V. 136. P. 571-602.

[20] Kiselman C. O. On entire functions of exponential type and indicators of analytic functions // Acta Math. 1967. V. 117. P. 1-35.

[21] Kronheimer P. B. Embedded surfaces and gauge theory in three and four dimensions // Preprint. Harvard, 1996.

[22] Kronheimer P. B., Mrowka T. The genus of embedded surfaces in the projective plane // Math. Res. Lett. 1994. V. 1. №6. P. 797-808.

[23] Kronheimer P.B., Mrowka T. Monopoles and contact structures // Invent. Math. 1997. V. 130. № 2. P. 209-255.

[24] Кружилин Н. Г. Двумерные сферы в границах строго псевдовыпуклых областей в $\mathbb{C}^{2} / /$ Изв. АН СССР. Сер. матем. 1991. Т. 55. С. 1194-1237.

[25] Lai H. F. Characteristic classes of real manifolds immersed in complex manifolds // Trans. Amer. Math. Soc. 1972. V. 172. P. 1-33.

[26] Lempert L. Algebraic approximation in analytic geometry // Invent. Math. 1995. V. 121. № 2. P. 335-354.

[27] Lisca P., Matić G. Tight contact structures and Seiberg-Witten invariants // Invent. Math. 1997. V. 129. № 3. P. 509-525.

[28] Мальгранж Б. Лекции по теории функций нескольких комплексных переменных. М.: Наука, 1969.

[29] Matsushima Y., Morimoto A. Sur certains espaces fibrés holomorphes sur une variété de Stein // Bull. Soc. Math. France. 1960. V. 88. P. 137-155.

[30] Moore J. D. Lectures on Seiberg-Witten Invariants // Lecture Notes in Math. V. 1629. Berlin: Springer-Verlag, 1996.

[31] Morgan J. W. The Seiberg-Witten Equations and Applications to the Topology of Smooth Four-Manifolds. Mathematical Notes. V. 44. Princeton: Princeton Univ. Press, 1996.

[32] Morgan J.W., Szabó Z., Taubes C.H. A product formula for Seiberg-Witten invariants and the generalized Thom conjecture // J. Differential Geom. 1996. V. 44. № 4 . P. 706-788. 
[33] Немировский С. Ю. Штейновы области на алгебраических многообразиях // Матем. заметки. 1996. Т. 60. С. 295-298.

[34] Немировский С. Ю. Голоморфные функции и вложенные вещественные поверхности // Матем. заметки. 1998. Т. 63. С. 599-606.

[35] Немировский С. Ю. О вложениях двумерной сферы в штейновы поверхности // Докл. PAH. 1998. T. 362. C. 442-444.

[36] Ozsváth P., Szabó Z. The symplectic Thom conjecture // Ann. of Math. (to appear).

[37] Rosay J. P. A counterexample related to Hartogs' phenomenon (a question by E. Chirka) // Michigan Math. J. 1998. V. 45. P. 529-535.

[38] Siu Y.-T. Every Stein subvariety admits a Stein neighbourhood // Invent. Math. 1976. V. 38. P. 89-100.

[39] Смирнов М. М., Чирка Е. М. О полиномиальной выпуклости некоторых множеств в $\mathbb{C}^{n} / /$ Матем. заметки. 1991. Т. 50. С. 81-89.

[40] Stout E. L. Algebraic domains in Stein manifolds // Banach Algebras and several complex variables. Proc. Conf. New Haven/Conn. 1983. Contemp. Math. V. 32, 1984. P. 259-266.

[41] Takeuchi A. Domaines pseudoconvexes infinis et la riemannienne dans un espace projectif // J. Math. Soc. Japan. 1964. V. 16. P. 159-181.

[42] Takeuchi A. Domaines pseudoconvexes sur les variétés kähleriennes // J. Math. Kyoto Univ. 1967. V. 6. P. 323-357.

[43] Ueda T. Pseudoconvex domains over Grassmann manifolds // J. Math. Kyoto Univ. 1980. V. 20. P. 391-394

[44] Witten E. Monopoles and four-manifolds // Math. Res. Lett. 1994. V. 1. P. 769-796.

Математический институт

Поступила в редакцию

им. В. А. Стеклова РАН

07.05.1999

E-mail: stefan@mccme.ru 